\title{
Biohormonal revitalization therapy from the perspective of biological regenerative medicine: the evaluation of premature menopause and andropause treatment outcomes in longitudinal cohort study
}

\begin{abstract}
Restoration of hormonal homeostasis and, as a consequence, the improvements in well-being, performance status and sexual function of the individuals has always been an indispensable aspect of anti-aging medicine. Albeit hormone replacement therapy (HRT) being the easiest and most preferred therapeutic method employed by majority of physicians, the undisputed risks associated with HRT dictate the necessity to search for alternative treatment solutions, capable of pledging nearly the same efficacy with considerably superior safety. In this regard, optimization of endogenous hormone synthesis and expression via the stimulation of hypothalamo-hypophyseal-adrenal axis with cell therapy beckons our attention as biological doctors. The current paper offers description of treatment protocols and analysis of the outcomes of biohormonal rejuvenation and revitalization by the means of cell therapy.
\end{abstract}

Keywords: regenerative medicine, cell therapy, menopause, andropause, biohormonal therapy, revitalization, stem cells, biological medicine
Volume 10 Issue 4 - 2019

\author{
Dmytro Klokol, ${ }^{1-4}$ Mike KS Chan, ${ }^{1-4}$ \\ Lingeswran Nallenthiran,' Michelle BF \\ Wong, ${ }^{1,2,4}$ Volodymyr Chernykh, ${ }^{1,2,3}$ Simon \\ Yefimov, ${ }^{1,2,3}$ Yuriy Nalapko, ${ }^{1,3}$ Bawani \\ Nesamany' \\ 'European Wellness Academy (Germany,Asia-Pacific), Germany \\ ${ }^{2}$ Stellar Biomolecular Research and Innovations, Germany \\ ${ }^{3}$ European Wellness Clinics International group, Germany \\ ${ }^{4} \mathrm{FCTI}$ (Asia-Pacific), Germany
}

\section{Correspondence: Dmytro Klokol, European Wellness} Academy (Germany, Asia-Pacific), Stellar Biomolecular Research and Innovations (Germany), European Wellness Clinics International group, Germany, Email dr.dmyro@sbi-europe.com

\section{Introduction}

Nature would prove that aging is inexorable. As age advances, the degenerative changes and shifts in bodily biochemical regulations are becoming more pronounced affecting performance status and quality of life. However, aging disproportionate to timeline is uncalled for and leads to several predicaments. Although being an expected biological phenomenon integral to the aging process, the deterioration of sex hormone equilibrium and decline of the reproductive function are often stumbled upon in a much younger age, which from the point of view of human physiology, psychology and sociology is considered as reproductive. Premature decline in reproductive function is known to affect approximately $2 \%$ of the female population and at least $7 \%$ of the male population in the reproductive age. ${ }^{1,2}$ Premature menopause is characterised by increased serum gonadotropin levels corresponding to the low levels of estrogen with reduced physiological functions of the ovary earlier than the mean menopausal age for a given population. ${ }^{1,3}$ Premature aging can be attributed to several factors including certain infection, environmental and genetic factors, underlying medical conditions, iatrogenic causes and most certainly lifestyle.

Premature menopause mimics the exact signs and symptoms of menopause such as irregular menses, hot flushes, mood swings, atrophic vaginal changes, decreased sexual drive, imbalance of the sleep cycle and emotional and psychological turmoil.

A serum estradiol level of $30 \mathrm{pg} / \mathrm{ml}$ or less with concurrent elevation of Follicle Stimulating Hormone (FSH) level beyond $40 \mathrm{mIU} / \mathrm{ml}$ is diagnostic of premature menopause. Osteoporosis, infertility, increased risk of cardio and cerebrovascular diseases and premature deaths are among the leading complications of premature menopause. $^{1-4}$

The clinical pattern of the onset of andropause is very much different from that of menopause. Gradual onset, it is estimated that the testosterone production drops by approximately $1-2 \%$ per year in males after the age of thirty. Factors influencing this rate include the underlying chronic illnesses, lifestyle, environmental and emotional health and iatrogenic causes. Premature andropause may lead to erectile dysfunction and decreased sexual drive, endocrine disorders such as diabetes mellitus and dyslipidemia, depression, obesity and musculoskeletal disorders with osteoporosis and marked sarcopenia being more common., ${ }^{2,56}$

It is important to acknowledge that factors contributing to the onset and rapid development of premature menopause and andropause include but not limited to chronic stress, low-cholesterol intake and decline in cholesterol biosynthesis, chronic systemic inflammation, lifestyle, etc. Increased Body Mass Index, low physical activity, use of tobacco and alcohol, genetic predisposition to early menopause or andropause, consumption of polyunsaturated fat, nulliparity and chastity are known to promote premature hormonal changes. On the other hand, overstress resulting in the phenomenon of pregnenolone steal, overexpression of pro-inflammatory markers, increased level of Sex Hormone Binding Globulin (SHBG) also contribute to reduction of bioavailable sex hormones, hence worsen the clinical manifestations of premature menopause and andropause. 


\section{Conventional management of premature menopause and andropause}

Hormone replacement therapy has been the mainstay of the treatment modality for premature menopause and andropause. This involves supplementing the deficient hormones, estradiol, estriol and progesterone. By any stretch of the imagination, hormone replacement has proven to successfully alleviate the relevant symptoms, being expressly effective in regeneration of skeletal muscle, prevention of osteoporosis, better cognitive ability and improvement of menopausal symptoms. ${ }^{7,8}$ Despite the desired outcomes, research has proven the pitfall of hormonal replacement. Increased risk of breast cancer has been documented among women who underwent HRT, as reported by Women's Health Initiative Trial ${ }^{9}$ and The Million Women Study (Beral and Million women Study Collaborators, 2003). The Breakthrough Generation Study which was carried out from 2003 to 2015 recruited 113693 women to determine the side effects of the HRT. The study concluded that women who undergo hormone replacement for 10 years or more are 3.5 times more likely to suffer from breast cancer compared to those who weren't treated with hormones. A large placebo controlled Heart and Estrogen/Progesterone Replacement Study (HERS) prove that HRT increases the risk of coronary artery disease by $50 \%$ within the first year of usage. Many other similar clinical trials have shown an increased risk of coronary artery disease between 1.4 to 1.8 times with short term usage of estrogen based hormone replacement therapy. A different HERS reported 3.9 fold increased risk in thromboembolic event among women who took hormone replacement therapy. Latest data confirms the increased risk of stroke and venous thromboembolism and long term risk of breast cancer with standard dose of hormone replacement therapy. ${ }^{10,11}$ A Postmenopausal Estrogen/Progesterone Interventions (PEPI) study reported an increased risk of breast cancer and also endometrial hyperplasia, rendering a precancerous condition among some women at the rate of $10 \%$ a year. ${ }^{9}$

The correlation between decreased serum testosterone level and mortality in long term has been established well ahead. Testosterone replacement has proven to decrease mortality rate among treated group $(8.4 \%)$ compared to the control group, $19.2 \%$. Studies have also suggested that the survival rate of men with type 2 diabetes mellitus is higher among men who have been treated with testosterone replacement. Multiple studies have shown the beneficial effect of testosterone replacement among patients who suffer from metabolic syndrome, type 2 diabetes mellitus and even anemia. ${ }^{11}$ Affirmative outcomes have been recorded among patients receiving testosterone replacement therapy to treat HIV infection related androgen deficiency. ${ }^{12}$ Testosterone replacement therapy has been successfully used to treat hypogonadism, in turn improving sex drive, cognitive abilities and cardiovascular health. Other similar trials documented promising outcome of testosterone replacement therapy to treat anxiety, depression and psychological stress.

Several side effects of testosterone HRT have been reported. This includes elevated blood pressure, ${ }^{13}$ cardiac hypertrophy and modulation of cardiomyocytes. ${ }^{13,14}$

\section{Novel approach in management of premature menopause and andropause}

The advent of novel therapeutic modalities such as gene therapy, cell therapy and other biological therapies has made it possible to successfully treat various disorders including endocrinopathies.
Hormonal insufficiency is basically caused by the primary organs involved or due to hypopituitarism, keeping other causes aside. Decades of research have proven that beyond the shadow of the doubt cell therapy has the capability to regenerate and rejuvenate secretory cells and in turn stimulate and regulate the endocrine system. ${ }^{15-17}$ The regulation of primary endocrine organs via the hypothalamohypophyseal axis has been described by Guillemin and Rosenberg (1955). This opened up the pedestal to experiment the effects cell therapy on hypothalamus and the pituitary glands and its regulatory effects on the primary endocrine organs. Flipping back the history of cell therapy would give an account of successful cell therapy of anterior pituitary gland in treating primary amenorrhea, testicular peptides for male revitalization, and ovarian peptides for dysmenorrhea and pituitary cell extracts for diabetes insipidus. ${ }^{18}$

The far fetching achievements of the biological doctors in treating the endocrine disorders with cellular therapy paved the path to describe the usage of organ specific peptide therapy in prevention and treatment of premature menopause and andropause by direct gonadal stimulation in turn reversing the dyshormonal bodily changes due to these conditions. Apart from direct stimulatory effect of targeted cell therapy protocols on the function of endocrine organs, cell therapy reduces chronic inflammation and optimizes systemic metabolism that results in concomitant beneficial influence on well-being in general and reproductive system in particular. ${ }^{17,19,20}$

Each case of hormonal disorders should be approached individually. Depending on the patient's biochemical profile, medical history and severity of hormonal disturbances various tools from the cell therapy's arsenal may be used. In our busy international clinical practice we prefer a multi-step multimodal therapeutic tactic. A session of autologous or allogeneic cell therapy with MSCs is done on the initial stages in order to prepare patient for the targeted organ-specific cell therapy. Although MSCs neither home to endocrine organs nor directly stimulate their function, positive effects are achieved via the reduction of the pro-inflammatory markers and optimization of patient's inner biological terrain. Main component of the biohormonal treatment protocol is represented by one of the organ-specific cell therapy products-either precursor stem cells (FCTI Inc.) or Frozen Organo Cryogenics (SBI, Germany) or even products containing subcellular ingredients id est cell extracts and peptides (SBI, MFplus).

To ensure that cell therapy works best we consider pregnenolone supplementation as an essential component of biohormonal therapy. In number of cases we find reasonable to additionally administer DHEA as well.

In our previous works we described the mechanisms through which cell therapy is capable of modulating the endocrine function and restoring the functional activity of the organs and systems undergoing the process of degeneration. ${ }^{15,18,21}$ We have also proven, on the results of the pilot observational studies done in the past, the potential efficacy of cell therapy in reversal of premature menopause and andropause. . $^{1720,21}$

\section{Objective of the study}

This particular study was designed to determine the efficacy of biohormonal therapy using combination of organ-specific cell extractspeptides (Mito Organelle, MF+, Germany) from hypothalamus, pituitary gland, adrenals, ovaries/testicles (gender specific) and liver in the management of premature menopause and andropause. The study was a part of the multicentric multinational research project endorsed 
by International Board for Aesthetic \& Regenerative Medicine (USA) and Scientific German Society for Biomolecular Medicine Research (Germany).

\section{Materials and methods of the study}

The observed group consisted of 26 men (age 34-53y.o.; mean age-42y.o.) and 29 women (age 29-47y.o.; mean age-38.5y.o.).

The inclusion criteria in the male group were three or more clinical symptoms of andropause (hypogonadism) confirmed by reduction of serum total testosterone level. The normal range of total testosterone level in males is arguably considered to range within 300 to $1000 \mathrm{ng} /$ dl. The optimal level of total testosterone is accepted to be $700 \mathrm{ng} / \mathrm{dl}$. In our global practice we consider levels of total testosterone in males $0-550 \mathrm{ng} / \mathrm{dl}$ as pathological and use it as a biochemical indication of premature andropause. ${ }^{22}$

The inclusion criteria for female subjects were three or more clinical symptoms of premature menopause co-existing with reduction of serum estradiol level below 40pg/ml and elevated FSH levels above $40 \mathrm{mIU} / \mathrm{ml}$. The optimal estradiol level on the $7^{\text {th }}$ day of the menstrual cycle in premenopausal females was accepted as $65-90 \mathrm{pg} / \mathrm{ml} .^{23,24}$

Sex hormone binding globulin (SHBG) is routinely checked in all our patients and Free Androgen Index (FAI) is calculated. A standard widely accepted chemiluminescent microparticle immunoassay method was used for total testosterone, estradiol and SHBG assay. Levels of free testosterone, percentage of free testosterone and bioavailable testosterone were routinely calculated for all observed male subjects. ${ }^{25} \mathrm{~A}$ complete hormonal profiling is done to all of the patients in European Wellness clinical facilities as a part of anti-aging screening. Apart from already mentioned total testosterone, SHBG, estradiol levels and FSH (for females), it includes but not limited to: serum progesterone, luteinizing hormone, dehydroepiandrosterone sulphate (DHEA), cortisol, thyroid function screening, insulin and insulin-like growth factor-1.

Following the baseline biochemical investigations and commencement of the treatment protocol clinical re-evaluation of the symptoms and laboratory tests were repeated upon the completion of the treatment (after 4 months), after 6 months and after 12 months.

The exclusion criteria were: premature menopause or andropause due to chromosomal abnormalities, surgical removal of gonads, pelvic tuberculosis, and history of radiotherapy or drug-induced ovarian or testicular suppression.

Male subjects received peptide therapy in a form of combination of extracts from hypothalamus, pituitary gland, adrenal cortex, testis and liver, commonly known as Male Fertility formulation 5-in-1 (Mito Organelles ${ }^{\mathrm{TM}}, \mathrm{MF}+$ ). Female subjects received peptide therapy in a form of combination of extracts from hypothalamus, pituitary gland, adrenal cortex, ovaries and liver, known under the code name of Female Fertility formulation 5-in-1 (Mito Organelles ${ }^{\mathrm{TM}}, \mathrm{MF}+$ ). The above mentioned cell therapy products were administered intramuscular $(2.5 \mathrm{ml})$ thrice per week with total duration of 4 months.

Concurrent supplementation with Pregnenolone in the dose 50 or $100 \mathrm{mg}$ and selective supplementation with DHEA in either oral form or absorbable cream is an integral part of our generally accepted clinical paradigms in cases when we encounter the phenomenon of pregnenolone steal, hormonal disorders or general anti-aging practice. The administration of necessary supplements was continued after the completion of biohormonal cell therapy protocol and throughout the entire period of follow up. A compulsory condition for the study recruitment was exclusion of all other forms of HRT from the treatment given to the study subjects.

After the completion of protocol clinical symptoms were reevaluated. Laboratory hormone level investigations were repeated upon completion of the treatment protocol, 3 months and 1 year after the end of biohormonal cell therapy. There were no patients dropped out of the study.

\section{Obtained results of the study}

As it was anticipated from the patient's complains and clinical manifestations, practically all patients had pronounced biochemical alterations. Majority of patients in both groups had increased level of SHBG, ferritin, and homocysteine, low level of IGF-1 and avid biomarkers of metabolic syndrome.

Within the first two to three weeks since the start of the biohormonal treatment protocol most of the observed patients have reported a subjective improvement of their physical condition, predominantly in terms of reduction of the chief complains related to the menopause or andropause. Practically all patients experienced increase in mental alertness and performance status, reduction of fatigue, improvements of sleep quality, memory and mood.

The levels of total testosterone in the male subgroup on the $D 0$ were scattered between $187 \mathrm{ng} / \mathrm{dl}$ as lowest detected total testosterone level and $392 \mathrm{ng} / \mathrm{dl}$ being the highest detected level. The mean baseline total testosterone level varied in the range between 279 to $294 \mathrm{ng} /$ $\mathrm{dl}$ (Chart 1). The level of SHBG was above the normal range in all of the observed patients and widely varied between $52.5 \mathrm{nmol} / \mathrm{L}$ and $167.2 \mathrm{nmol} / \mathrm{L}$. As a result of such changes in biochemical parameters the calculated values id est FAI and percentage of bioavailable testosterone were grossly deranged in predominant majority of the observed male patients.

Shortly after the commencement of the biohormonal treatment protocol most of the patients noticed a dramatic shift in the level of their well-being, performance status and had noticeable improvements in clinical symptoms related to their andropause. All observed men noted significant increase of libido, improvement of sexual function, increase of energy level and better physical performance. Four months after the commencement of the biohormonal therapy the levels of total testosterone in some cases increased by nearly $100 \%$ from the baseline and mean total testosterone level on D120 varied from 490 to $506 \mathrm{ng} / \mathrm{dl}(\mathrm{p}<0.05)$.

Further follow up of the patients has shown stability in the achieved clinical results and in hormonal status of the observed patients. Laboratory investigations done during the follow up on the D210 revealed that mean total testosterone level was in the range 552 to $575 \mathrm{ng} / \mathrm{dl}$, with lowest detected level being $390 \mathrm{ng} / \mathrm{dl}$ and highest$598 \mathrm{ng} / \mathrm{dl}$. One year after the biohormonal cell therapy mean level of total testosterone still demonstrated a notable stability though slightly reduced and varied between 462-483ng/dl (Chart 1).

Among other remarkable positive outcomes of our treatment protocol we have observed the improvements in SHBG assay, increase of FAI and the percentage of bioavailable testosterone. It also coincided with reduction of markers of systemic chronic inflammation (ferritin, homocysteine), increment of DHEA and insulin-like growth factor-1. 


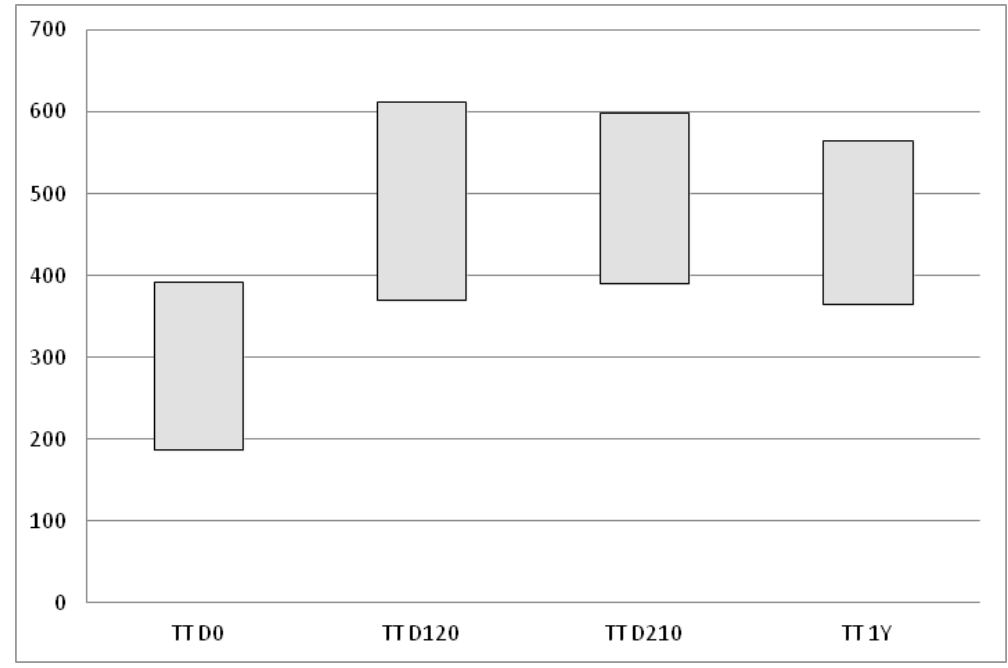

Chart I Changes of total testosterone (TT) mean level in the observed males measured in ng/dl.TT D0-baseline levels of total testosterone;TT DI20-levels of total testosterone upon completion of treatment protocol;TT D2 I0-levels of total testosterone 3 months after the completion of biohormonal therapy; TT IY-levels of total testosterone I year after the completion of treatment protocol $(\mathrm{N}=26)$.

Upon initial presentation the basal level of serum estradiol in the female subgroup varied from 9 to $32 \mathrm{pg} / \mathrm{ml}$, with the mean level ranging between 17.0 to $20.5 \mathrm{pg} / \mathrm{ml}(19.5 \pm 0.5 \mathrm{pg} / \mathrm{ml})$. At the same time the baseline FSH level varied from 45 to $163 \mathrm{mlU} / \mathrm{ml}$, with mean FSH level being $65.9 \pm 0.4 \mathrm{mlU} / \mathrm{ml}$. In the end of the 4 th month of biohormonal therapy with organ peptides Mito Organelles ${ }^{\mathrm{TM}}(\mathrm{MF}+)$ most of the patients in the female group (24 out of 29) had reversal or at least significant improvements in the status of their well-being and clinical manifestations. The rest five women had experienced some positive changes in terms of improvement of menopausal symptoms, however not significant and profound enough to be accepted as a significant clinical improvement. The cases with least of clinical improvements were associated with minimal shifts in FSH and estradiol assays. In the majority of female subjects' (24 out of 29) clinical progress positively correlated with positive changes in their hormonal profile. Thus, serum estradiol level on D120 ranged $45-52 \mathrm{pg} / \mathrm{ml}$ (mean serum estradiol level $48.5 \pm 0.5 \mathrm{pg} / \mathrm{ml} ; \mathrm{p}<0.05$ ) and serum FSH level on the average varied from 35 to 42 (mean serum FSH $37.5 \pm 0.5 \mathrm{mlU} / \mathrm{ml} ; \mathrm{p}<0.05)$ (Chart 2) (Chart 3).

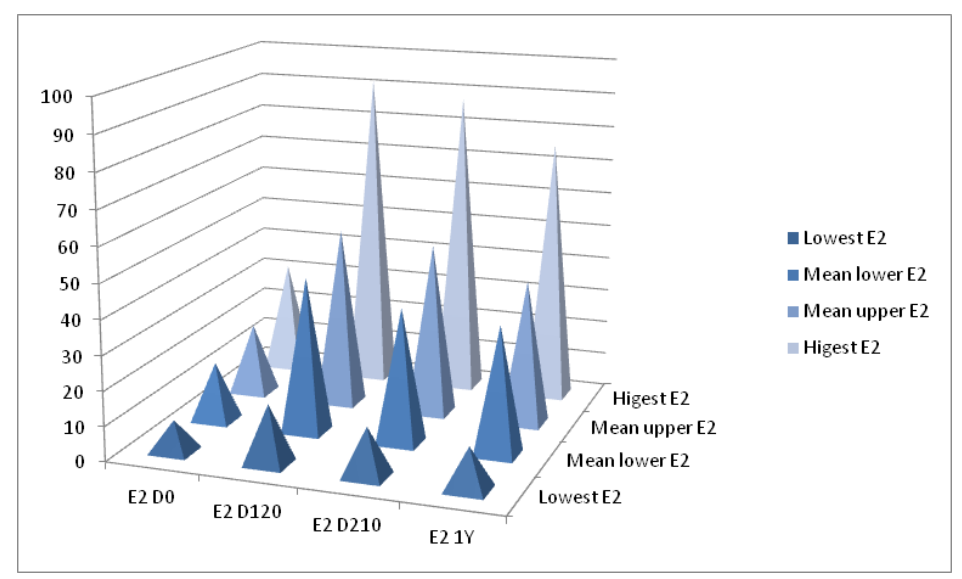

Chart 2 Changes in estradiol (E2) mean level in the observed females measured in ng/dl. E2 D0-baseline levels of estradiol; E2 DI20-levels of estradiol upon completion of treatment protocol; E2 D2I0-levels of estradiol 3 months after the completion of biohormonal therapy; E2 IY-levels of estradiol I year after the completion of treatment protocol (N-29).

Hormonal screening of female subjects on D210 and after one year post completion of biohormonal cell therapy treatment protocol is illustrated by Chart 2 and Chart 3. On D210 mean estradiol levels ranged between $39-50 \mathrm{pg} / \mathrm{ml}, \mathrm{FSH}-37-45 \mathrm{mlU} / \mathrm{ml}$. One year after completion of biohormonal protocol mean estradiol was between 37 to $42 \mathrm{pg} / \mathrm{ml}$; FSH- $40-47 \mathrm{mlU} / \mathrm{ml}$. 


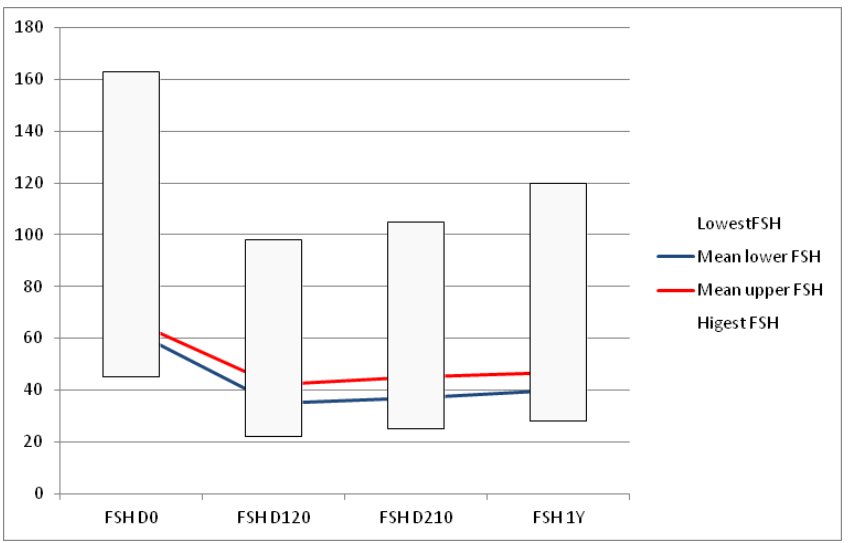

Chart 3 Changes in FSH mean level in the observed females measured in ng/dl. FSH D0-baseline FSH levels; FSH DI20-FSH levels upon completion of treatment protocol; E2 D2 I0-FSH levels 3 months after the completion of biohormonal therapy; FSH IY-FSH levels I year after the completion of treatment protocol $(\mathrm{N}=29)$.

\section{Discussion}

Indeed, premature menopause and andropause present a particular challenge for medical practitioner. HRT possesses substantial risks that gradually increase with the duration of the therapy received. The main concern of medical researchers is to reduce those risks. Biohormonal therapy with precursor stem cells or organ-specific cell extracts is based on modulation of endocrine function along the hypothalamohypopheseal-adrenal axis and past experience of such therapies has proven its potential. ${ }^{15-17,19-21}$ The treatment protocol discussed in this paper includes combination of extracts from hypothalamus, pituitary gland, adrenal cortex, gonads and liver (Mito Organelles ${ }^{\mathrm{TM}}$, MF+, Germany) and addresses to all levels of sexual hormones biosynthesis by modulating the function of the targeted endocrine organs. The current prospective longitudinal study has proven that four months of biohormonal cell therapy targeting endocrine function in individuals with premature menopause and andropause have provided dramatic improvement of symptoms and normalization of hormonal profile in $82 \%$ of women. The key to success in biohormonal revitalization and reversal of premature menopause lies in its early detection and timely commenced a proper treatment protocol, which should include administration of precursors of steroid hormones and stimulation of endogenous hormonal biosynthesis by the means of cell therapy.

Biohormonal cell-based therapy promises even higher success rate in male category of patients, which with the appropriate diagnostic and therapeutic approach can even bestow close to a $100 \%$ attainment rate. It is crucially important to simultaneously apply therapeutic efforts targeting the amelioration of systemic inflammation, metabolic syndrome and cellular senescence. ${ }^{21}$

\section{Conclusion}

The current study has proven to successfully improve the gonadal function and reverse the premature menopause and andropause state, using the paradigms of biological regenerative medicine and its main therapeutic tool-cell therapy. This study has to be expanded to a larger scale with greater number of subjects to ensure reliability and reproducibility of the results. A longitudinal follow up is necessary to evaluate the long term effect and establish the long-term safety profile of the modality.

\section{Acknowledgments}

None.

\section{Conflicts of interest}

Authors disclose no conflict of interests in publication of this study.

\section{References}

1. Ceylan B, Özerdoğan N. Factors affecting age of onset of menopause and determination of quality of life in menopause. Turk $J$ Obstet Gynecol. 2015;12(1):43-49.

2. Singh P. Andropause: current concepts. Endocrinol Metab. 2013;17(Suppl 3):S621-S629.

3. Shuster LT, Rhodes DJ, Gostout BS. Premature menopause or early menopause: long long-term health consequences. Maturitas. 2010;65(2):161-166.

4. Dorfman RI. Estrogen. In: McGraw-Hill. 10th ed, Encyclopedia of Science \& Technology; 2007:682-685.

5. Bansal VP. Andropause. A clinical entity. Journal of Universal College of Medical sciences. 2013; 1(2):54-68.

6. Nieschlag E, Behre HM, Nieschlag S. Testosterone: action, deficiency, substitution. 3rd ed, Cambridge: Cambridge University Press; 2004.

7. Barret-Connor E, Stuenkel CA. Hormone replacement therapy (HRT) risks and benefits. Int Jounr Epidemiol. 2001;30(3):423-426.

8. Velders M, Diel P. How sex hormones promote skeletal muscle regeneration. Sports Med. 2013;43(11):1089-1100.

9. Rossouw JE, Manson JA, Kaunitz AM, et al. Lessons learned from the women's health initiative trials of menopausal hormone therapy. Obstet Gynecol. 2013;121(1):172-176.

10. Jones ME, Schoemaker MJ, Wright L, et al. Menopausal hormone therapy and breast cancer: what is the true size of the increased risk? Br J Cancer. 2016;115(5):607-615

11. Yang D, Li J, Yuan Z, et al. Effect of hormone replacement therapy on cardiovascular outcomes: a meta-analysis of randomized controlled trials. PLOS ONE. 2013;8(5):e62329.

12. Blick G, Khera M, Bhattacharya RK, et al. Testosterone replacement

Citation: Klokol D, Chan MKS, Nallenthiran L, et al. Biohormonal revitalization therapy from the perspective of biological regenerative medicine: the evaluation of premature menopause and andropause treatment outcomes in longitudinal cohort study. Obstet Gynecol Int J. 20I 9; I0(4):236-24I. 
therapy in men with hypogonadism and HIV/AIDS: results from the TRiUSregistry. Postgrad Med. 2013;125(2):19-29.

13. He J, Bhasin S, Binder EF, et al. Cardiometabolic risks during anabolic hormone supplementation in older men. Obesity. 2013;21(5):968-975.

14. Marsh JD, Lehmann MH, Ritchie RH, et al. Androgen receptors mediate hypertrophy in cardiac myocytes. Circulation. 1998;98(3):256-261.

15. Klokol D, Nallenthiran L, Chan MKS, et al. Cell therapy as the main stratagem of anti-aging and regenerative medicine. Europ J Pharm Med Res. 2019;6(6):295-299.

16. Nasli Esfahani E, Ghavamzadeh A, Larijani B. Therapeutic uses of stem cells in endocrinology- review article. Iranian J Publ Health. 2014;43(1):35-48.

17. Chan MKS, Wong MBF, Klokol D, et al. Biohormonal therapy in early management of premature menopause and andropause. Internat Journ Cur Med Pharm Res. 2017;3(1):1278-1281.

18. Schmid F, Stein J. Cell research and cellular therapy. Switzerland: OttPubl House; 1967.

19. Klokol D. Mitochondrial specific peptides in anti-aging and therapeutic rejuvenation: An innovative fusion of mitochondrial medicine and cellular therapy. Aesthet Dermatol and Surg. 2016;5-CC:35-36.
20. Klokol D. HRT-free management of premature andropause with advanced organ-specific peptide therapy. J Nephrol Ther. 2017;7(4):38.

21. Klokol D, Chan MTKS. Stem cells in regenerative medicine: carpe diem, carpe vitum. UK: Troubador; 2019. 495 p.

22. Travison TG, Vesper HW, Orwoll E, et al. Harmonized reference ranges for circulating testosterone levels in men of four cohort studies in the United States and Europe. J Clin Endocrinol Metab. 2017;102(4):1161-1173.

23. Barbieri RL. The endocrinology of the menstrual cycle. Methods Mol Biol. 2014;1154:145-169.

24. Pouresmaeili F, Fazeli Z. Premature ovarian failure: a critical condition in the reproductive potential with various genetic causes. Int $J$ Fertil Steril. 2014;8(1):1-12.

25. Veldhuis JD, Bondar OP, Dyer RB, et al. Immunological and mass spectrometric assays of SHBG: consistent and inconsistent metabolic associations in healthy men. J Clin Endocrinol Metab. 2014;99(1):184 193. 